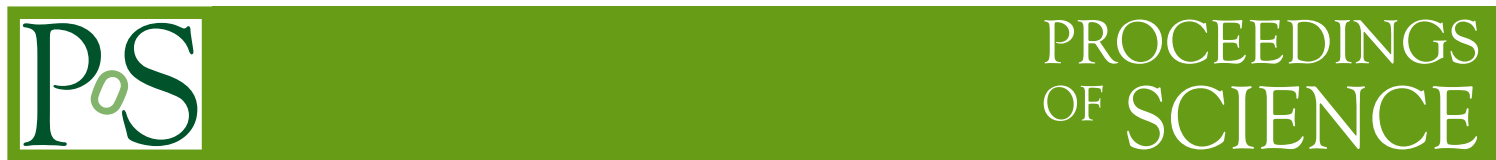

\title{
Cosmic censorship revisited from a quantum mechanical standpoint
}

\author{
N. Pappas* \\ National and Kapodistrian University of Athens \\ E-mail: npappasecc.uoi.gr
}

\begin{abstract}
The cosmic censorship hypothesis $(\mathrm{CCH})$ has the key role of ensuring that naked singularities never do emerge in the context of classical general relativity based on the demand that an event horizon should always develop around a singularity prohibiting the direct interaction of the latter with the rest of the Universe. This is a very important function in order for general relativity to be self-consistent. Even today, more than 4 decades after its formulation the origin and the validity range of the hypothesis remain open questions. Here both the weak and the strong version of $\mathrm{CCH}$ are examined under the light of Heisenberg's uncertainty principle. This approach dictates then that the emergence of an event horizon that shields the singularities from observers at infinity is also quantum mechanically favored. Further down that road, however, the same approach indicates that singularities never actually form in the usual sense, thus no naked singularity danger exists in the first place, rendering $\mathrm{CCH}$ redundant.
\end{abstract}

18th International Conference From the Planck Scale to the Electroweak Scale 25-29 May 2015

Ioannina, Greece

\footnotetext{
* Speaker.
} 


\section{Introduction}

Singularities as space-time regions, where curvature invariant quantities (like $R_{\mu v \rho \sigma} R^{\mu v \rho \sigma}$ ) deviate, are perhaps the most problematic notions in Physics. It is only sufficient to point out that we describe the singular regions through the space-time metric even though, strictly speaking, these regions should not be considered as part of that space-time since the metric there is ill-behaved. To deal with the apparent incoherence one moves on to consider such singular points as ideal boundary points attached to the ordinary, well-behaved manifold in the context of an augmented space-time. Naked singularities are deeply puzzling objects and a source of lawlessness since, by definition, the space-time structure breaks down there while, at the same time, physical laws presuppose space and time to develop and manifest themselves. However, against all expectations, Hawking and Penrose have shown that the emergence of singularities is inevitable in a very large class of Universe types including ours [1]. In order to deal with the challenges imposed by their existence Penrose put forward the famous cosmic censorship hypothesis $(\mathrm{CCH})$ [2]. In the years to come the original hypothesis was further developed and reformulated into two versions. The weak version (wCCH) states that a singularity is at every moment surrounded by an absolute event horizon for observers at infinity so they can never directly see it. The strong version ( $\mathrm{sCCH})$, on the other hand, states that no observer at any time or place can have any direct interaction with a singularity [3]. Thanks to cosmic censorship then a naked singularity should never occur except perhaps in the case of some special matter distributions which are not expected to occur in a realistic Universe (for an extensive presentation of the issues concerning singularities and $\mathrm{CCH}$ see [4] [5] [6]).

Interestingly enough, despite its crucial role as described earlier, $\mathrm{CCH}$ does not stem from some well established physical law or mathematical theorem. Soon after it was proposed, it was declared as one of the most important open questions in classical general relativity [3], whose derivation remains obscure until nowadays (see [7] [8] for reviews on the work done sofar). It is a rather convenient and in a way necessary hypothesis, that considering the catastrophic impact of naked singularities in our perception of Nature, we gladly accept as (probably) true. At first, arguments supporting the idea were based largely on causality and geometry, usually expressed in terms of the TIF (terminal indecomposable future) and PIF (terminal indecomposable past) notions [9] (see again [6]). Penrose was able to derive solid inequalities involving black hole masses and horizon radii in support of his hypothesis [10], which were also shown to hold true in a series of different situations [11]. Moreover, $\mathrm{CCH}$ was proven to be valid in series of physically interesting, specific space-times [12]. Sceptics at the same time focused on the effort to construct counterexamples where naked singularities could actually emerge [13]. The majority of those examples, however, was based on considering very special and idealized conditions to hold - therefore least possible to exist in a realistic Universe - so the validity of $\mathrm{CCH}$ was never really threatened by them. Soon it was evident that classical arguments had reached their limitations and consequently a quantum treatment was in order. It was anticipated by many scientists that the inclusion of quantum mechanics in the picture of gravitational collapse could do away all our difficulties to cope with singularities. Besides, it is common belief that the problematic way we describe singularities represents much more our lack of understanding about the laws of quantum gravity that take over when radii of space-time curvature of the order of Planck length are in question, than their true nature. Quantum mechanics actually was proven very successful in resolving many of the thought 
experiment counterexamples in favor of $\mathrm{CCH}$. Famously, it was used to show that it is not possible to over-spin or over-charge a maximal Kerr black hole to produce a naked singularity [14] (a procedure first considered in [15]).

Pretty much in the same line of thinking we chose to engage quantum mechanics in the treatment of point-like singularities lying at a finite distance (as opposed to singularities lying at infinity or thunderbolts) [16]. The idea is to use Heisenberg's uncertainty principle,

$$
\Delta x \cdot \Delta p \geq 1 \quad \text { (in Planck units) }
$$

as basis for our approach, upgrading it to be treated as the most fundamental principle every natural system has to comply with, and then apply it to systems involving singularities to see the constraints it imposes concerning the properties of the latter.

\section{Rethinking weak censorship}

According to the provisions of general relativity, when too large a mass is concentrated in too small a volume unstoppable collapse of matter occurs which is expected to end up in the formation of a point-like singularity since no mechanism is know so far to be able to stop the aforementioned collapse. By definition, the size of the singularity goes to zero thus it occupies only a single point of space-time in the purest geometrical sense of the "point" notion. Having this in mind, in the case of a naked singularity, an observer effectively at infinity - practically at sufficiently large distance away from it to be in an asymptotically flat region - would, in principle, be able to determine its position with arbitrarily high accuracy by e.g. direct observation. However, the uncertainty principle states that when we determine the position of a quantum system with uncertainty $\Delta x \rightarrow 0$ we inevitably have to end up with complete lack of knowledge about its momentum $(\Delta p \rightarrow \infty)$, therefore about its energy as well. We argue, though, that this is not the case with naked singularities. Even though quantum gravity is necessary to describe the singularity per se, it is legitimate to expect that space-time at macroscopic distances away from the latter can be sufficiently accurately and reliably described by general relativity. In this context, it is always possible to experimentally "weigh" the singularity since it fundamentally is a gravitational object. E.g. by observing potential gravitational lensing effects or by measuring the trajectory, speed and acceleration of test bodies that get attracted by it etc. This way the mass/energy of the singularity would be known with uncertainty at most of the order of the mass itself evidently.

Combining all the above then means that the very existence of a naked singularity, apart from all other conceptual and physical puzzles that accompany it, would also lead to violations of the uncertainty principle. This totally unacceptable consequence is avoided thanks to the provisions of the wCCH. An event horizon of radius $r_{h} \sim M$, that exists in every kind of black hole type since it emerges because of the warping of the space-time continuum by the singularity mass itself, poses an unavoidable uncertainty regarding the actual position of the singularity of the order $\Delta x \sim r_{h}$, which is perfectly compatible with measuring its mass with $\Delta M \sim M$. In this perspective wCCH is not only necessary to make general relativity self-consistent, but it is also strongly quantummechanically favored to prevent uncertainty principle violations. 


\section{Rethinking strong censorship}

Let's move to examine sCCH under the same light. We consider a very large and massive system like the central region of a galaxy, where a trapped surface has already formed. Observers living on a planet within the trapped region will continue exist and naturally expect quantum mechanics to hold at all times until eventually they crash into the singularity, which will develop some time in their future. In this case, even though the singularity formed will always remain unseen by observers at infinity in accordance with the $\mathrm{wCCH}$ provisions, an observer inside the horizon will actually encounter a naked singularity while at a significantly large distance away from it. Since all arguments presented in the previous section hold also true for this observer a paradox rises. The need to resolve this paradox leads to the introduction of the sCCH which explicitly forbids an observer to interact with the singularity in any possible way no matter what the circumstance are. The main issue however is that since sCCH doesn't provide us with a mechanism capable of deterring this interaction, looks more like the ad hoc expression of a hope than a constraint imposed by some physical law or procedure.

A quite straightforward way out is to admit that the notion of unstoppable collapse is wrong - a byproduct of describing the whole process with the use of classical theories - and therefore no point-like singularity is formed at all. At Planck scale quantum effects should get so enhanced to counterbalance the gravitational contracting forces, stop the collapse and prevent singularities from forming in the way we conceive them to do today For example the confinement of matter in an ever decreasing volume, which means that it will acquire an ever increasing momentum/energy, according, once again to the uncertainty principle, to end up behaving like a highly energetic gas whose pressure will constantly grow to eventually stop the contraction is a plausible mechanism to be explored in a work to come.

The rather radical approach proposed that no singularity actually does form, is in accordance with a very interesting argument delivered by Geroch which deploys like this:

A) When a manifold admits a Cauchy surface (as it is the case for the majority of physically reasonable space-times), then it also admits a global time function $t$.

B) The latter increases along every future-oriented timelike curve, which can be chosen so that every $t=$ constant surface is a Cauchy one.

C) Cauchy surfaces cannot intersect the singularity and thus there is no time at which the singularity exists [17].

In conclusion, the revision of $\mathrm{CCH}$ with respect to the uncertainty principle lead to two interesting yet contradictory findings. First, we showed that wCCH should hold true also due to quantum mechanical reasons. On the other hand, since it is insufficient by itself to make the overall picture of a system containing singularities self-consistent, $\mathrm{sCCH}$ is necessary also to be valid. However, the restrictions imposed by the latter regarding the possibility of a direct interaction of an observer with the singularity are so strict that cannot actually be satisfied in all case where a singularity is formed. As a way out then one quite naturally makes the breakthrough to postulate that singularities never emerge in the usual sense, rendering all versions of the $\mathrm{CCH}$ redundant in the first place. 


\section{References}

[1] S. W. Hawking and R. Penrose, The singularities of gravitational collapse and cosmology, Proc. R. Soc. London A314 (1970) 529 and Gravitational collapse and space-time singularities Phys. Rev. Lett. A14 (1965) 57.

[2] R. Penrose, Gravitational Collapse: The Role of General Relativity, Revisita del Nuovo Cimento, Serie I 1 Numero Speciale (1969) 252.

[3] R. Penrose, Singularities of Space-Time in Theoretical Principles in Astrophysics and Relativity, University of Chicago Press, Chicago (1978) 217-243.

[4] R. P. Geroch, What is a Singularity in General Relativity?, Annals of Physics 48 (1968) 526.

[5] J. Earman, Bangs, Crunches, Whimpers and Shrieks, Oxford University Press (1995) 27-102.

[6] R. Penrose, The Question of Cosmic Censorship in Black holes and relativistic stars, University of Chicago Press, Chicago (1998) 103-122.

[7] R. M. Wald, Gravitational Collapse and Cosmic Censorship, [gr-qc/9710068]; T. P. Singh, Gravitational Collapse, Black Holes and Naked Singularities, [gr-qc/9805066].

[8] W. A. Hiscock, Magnetic charge, black holes, and cosmic censorship, Ann. Phys. (N. Y.) 131 (1981) 245; J. D. Bekenstein and C. Rosenzweig, Stability of the black hole horizon and the Landau ghost, Phys. Rev. D 50 (1994) 7239 [gr-qc/9406024] ; V. E. Hubeny, Overcharging a black hole and cosmic censorship, Phys. Rev. D 59 (1999) 064013 [gr-qc/9808043]; T. C. Quinn and R. M. Wald, Energy conservation for point particles undergoing radiation reaction, Phys. Rev. D 60 (1999) 064009 [gr-qc/9903014]; S. Hod, Black-hole polarization and cosmic censorship, Phys. Rev. D 60 (1999) 104031 [gr-qc/9907001] and Cosmic censorship: The Role of quantum gravity Gen.Rel.Grav. 32 (2000) 2333 [gr-qc/ 0011003 ].

[9] R. Geroch, E. H. Kronheimer and R. Penrose, Ideal points in space-time, Proc. R. Soc. London A347 (1972) 545.

[10] R. Penrose, Naked singularities, Ann. N.Y. Acad. Sci. 224 (1973) 125.

[11] R. Geroch, Energy Extraction*, Ann. N.Y. Acad. Sci. 224 (1973) 108; G. W. Gibbons, The time symmetric initial value problem for black holes, Commun. Math. Phys. 27 (1972) 87, Collapsing shells and the isoperimetric inequality for black holes, Class. Quant. Grav. 14 (1997) 2905 [hep-th/9701049] and in Global Riemannian Geometry, Ellis Horwood publications, Chichester (1984); P. S. Jang and R. M. Wald, The positive energy conjecture and the cosmic censor hypothesis, J. Math. Phys. 18 (1977) 41; G. Huisken and T. Ilmanen, A Note on Inverse Mean Curvature Flow, Int. Math. Res. Not. 20 (1997) 1045.

[12] A. Krolak, Towards the proof of the cosmic censorship hypothesis, Class. Quant. Grav. 3 (1986) 267, Towards the proof of the cosmic censorship hypothesis in cosmological space-times, J. Math. Phys. 28 (1987) 138 and Strong cosmic censorship and the strong curvature singularities, J. Math. Phys. 28 (1987) 2685; R. P. A. C. Newman, A theorem of cosmic censorship: a necessary and sufficient condition for future asymptotic predictability, Gen. Rel. Grav. 16 (1984) 175, Cosmic censorship and conformal transformations, Gen. Rel. Grav. 16 (1984) 943 and in Topological Properties and Global Structure of Space-Time, Plenum Press, New York (1986) 153-168; P. T. Chrusciel, J. Isenberg and V. Moncrief, Strong cosmic censorship in polarised Gowdy spacetimes, Class. Quant. Grav. 1 (1990) 1671. 
[13] K. S. Thorne, in Magic without magic, Freeman publications, San Francisco (1972); P. Yodzis, H.-J. Seifert and H. Muller zum Hagen, On the occurrence of naked singularities in general relativity, Commun. Math. Phys. 34 (1973) 135; P. Chrusciel, On space-times with U (1)E U (1) symmetric compact Cauchy surfaces, Ann. Phys. 202 (1990) 100; S. Shapiro and S. A. Teukolsky, Formation of naked singularities: the violation of cosmic censorship, Phys. Rev. Lett. 66 (1991) 994; M. Choptuik, Universality and scaling in gravitational collapse of a massless scalar field, Phys. Rev. Lett. 70 (1993) 9; D. Christodoulou, Examples of naked singularity formation in the gravitational collapse of a scalar field, Ann. Math. 140 (1994) 607; B. Steinmuller and J. P. Lasota, Radiating bodies and naked singularities, Phys. Lett. A 51 (1975) 191; K. Lake and C. Hellaby, Collapse of radiating fluid spheres, Phys. Rev. D 24 (1981) 3019; P. S. Joshi, in Global Aspects in Gravitation and Cosmology (chapter 7), Clarendon Press, Oxford (1993).

[14] S. Hod, Return of the quantum cosmic censor, Phys. Lett. B 668 (2008) 346 [gr-qc/0810 . 0079]; G. E. A. Matsas, M. Richartz, A. Saa, A. R. R. da Silva and D. A. T. Vanzella, Can quantum mechanics fool the cosmic censor?, Phys. Rev. D 79 (2009) 101502 [gr-qc/ 0905.1077 ]; S. Hod and T. Piran, Cosmic Censorship: The Role of Quantum Gravity, Gen. Rel. Grav. 32 (2000) 2333 [gr-qc/0011003].

[15] R. M. Wald, On perturbations of a Kerr black hole, J. Math. Phys. 14 (1973) 1453.

[16] N. Pappas A little quantum help for cosmic censorship and a step beyond all that Adv.High Energy Phys. 2013 (2013) 236974 [gr-qc/1312 . 3471].

[17] R. P. Geroch, Domain of Dependence, Journal of Mathematical Physics 11 (1970) 437. 